\title{
ASPECTOS SOCIO ECONÓMICOS DE LA PRODUCCIÓN Y COMERCIALIZACIÓN DE LA GRANADILLA (Pasiflora Ligularis Juss) EN OXAPAMPA
}

\section{SOCIO ECONOMIC ASPECTS OF THE PRODUCTION AND COMMERCIALIZATION OF THE PASAFLORA LIGULARIS JUSS IN OXAPAMPA}

\author{
Jorge Amaya Cubas ${ }^{6}$, Oscar Pérez Cabrera, Omar Galarza Leiva ${ }^{7}$
}

Facultad de Agronomía

\section{RESUMEN}

Consientes de las necesidades más sentidas de los pobladores de los anexos que conforman estos distritos y provincia de Oxapampa, se ha visto por conveniente realizar acciones para llevar adelante el presente proyecto y apoyar de esta manera el desarrollo socioeconómico de las organizaciones involucradas y que en base al cultivo de granadilla se fortalezca la economía comunal y poblacional, porque en comparación a la rentabilidad de otros productos que se producen en la zona como es el caso de maíz, frijol, yuca, plátanos, etc. La granadilla ofrece al nativo y colono una rentabilidad de hasta 10 veces más en comparación a los cultivos tradicionales, además, la producción es sumamente rápida y corta en comparación a otras plantaciones que demoran 3 a 5 años para iniciar la producción. La granadilla produce a partir de los 9 meses después de trasplantado y hasta los 7 años; la cosecha se realiza cada 15 días. Hemos dicho que el problema socio económico de las diferentes poblaciones, la baja rentabilidad de sus cultivos, obliga que se tenga que ver la forma que estos colonos y nativos puedan mejorar sus ingresos y por consiguiente su condición socio económico. El proyecto presenta dentro de su estructura un conjunto de información básica del ámbito del proyecto, sus áreas geográficas potenciales, el proceso productivo, los costos unitarios, rendimientos, rentabilidad, cronograma de ejecución.La concretización y puesta en marcha del presente proyecto coadyuvará a generar mayores ingresos que potencien la consolidación empresarial y una adecuada capitalización que tienda a mejorar los niveles de vida de los pobladores de la selva central sumidos en la actualidad en la miseria y la extrema pobreza. Se ha visitado lugares de estudios como Oxapampa, Chontabamba y Huancabamba, obteniéndose datos muy interesantes para la realización del proyecto.

Palabras Clave: costos unitarios, colonos.

\section{ABSTRACT}

Due to the awareness of the most pressing needs of the inhabitants of the annexes that make up the districts and province of Oxapampa, appropriate actions are proposed to carry out through this project to support the socioeconomic development of the organizations and cultures within these communal economies and populations. As compared to the profitability of products that are produced in the area such as corn, beans, cassava, bananas, etc, the passionflower gives the native farmer even higher profitability, up to 10 times more compared to traditional crops. In addition, production is extremely rapid compared to other crops that take 3 to 5 years to start production. The passionflower produces after 9 months of being transplanted and up to 7 years, and the harvest is done every 15 days. The problem of different socioeconomic populations, the low profitability of their crops, encourages then these settlers and

\footnotetext{
6 jamaya100@hotmail.com

7 ogale551@hotmail.com
} 
natives to improve their income and therefore their socioeconomic status. The project has within its structure a set of basic information on the scope of the project, its potential geographic areas, the production process, unit costs, performance, cost, implementation schedule. The realization and implementation of this project will contribute to generate more revenue to enhance business consolidation and adequate capitalization which will tend to improve the living standards of residents in the central jungle now mired in poverty and extreme poverty. Oxapampa Chontabamba and Huancabamba were visited yielding very interesting data for the project.

Key words: unit costs, settlers

\section{INTRODUCCION}

En el caso de la agricultura y agroindustria de exportación, por su efecto dinámico y multiplicador, ésta es considerada un medio para incrementar los ingresos del país. La granadilla, junto con la maracuyá, pertenece a la familia de las pasifloráceas y se le conoce con ese nombre en Centro y Sud América (Perú, Bolivia, Costa

Rica, Ecuador, Colombia, México y Guatemala), aunque en Hawai, Venezuela y Jamaica se le conoce como water lemon, granadilla de China o parchita amarilla, y granadilla, respectivamente. Su nombre científico es "Pasiflora ligularis" y es una planta trepadora que provee de una fruta ovoide 0 elíptica con un diámetro aproximado de 8 centímetros. Esta fruta crece sostenida a la vid por un pedúnculo largo que mide entre 6 y 12 centímetros de largo. Se caracteriza por su agradable sabor dulce, su agradable aroma y por tener una cáscara dura que le da cierta ventaja. Dentro de esta cáscara, se encuentra una pulpa cubierta por un saco membranoso y esponjoso. Esta pulpa es gelatinosa y contiene un promedio de 250 pequeñas semillas planas, elípticas, de color café oscuro o negro, envueltas por un arillo jugoso, transparente, dulce y aromático que constituye la parte comestible del fruto. Esta fruta viene siendo cultivada en las regiones de Pasco, Huánuco, Puno, Junín, Cajamarca, La Libertad y Piura.

Los objetivos fueron: Analizar los impactos socioeconómicos del cultivo de la granadilla en la zona. Estudiar el sistema de comercialización de este frutal y las normas que deben cumplir como producto de exportación.

Jiménez (2006). Menciona que las pasifloráceas mayormente son plantas trepadoras, para lo cual se hallan provistos de zarcillos, con flores complejas en los cuales se destaca un órgano típico y que la corona está formado por sépalos.

La granadilla es una planta oriunda del Perú, es una planta trepadora, pertenece a una gran variedad de especies de la familia Pasiflora.

Su uso es tanto medicinal como alimenticio pues combate inflamaciones gastrointestinales, es tenífugo, antihelmíntico, diurético.

La granadilla es la más comercializada en el Perú; su centro de producción está en los valle interandinos a altura entre los 900 a $2700 \mathrm{msnm}$ y en especial en los departamentos de Lima y La Libertad, también se cultiva en el departamento de Junín; Ancash, Huánuco, Ayacucho, Cuzco (Urubamba) y Cajamarca.

Es una trepadora vigorosa de tallos cilíndricos, hojas grandes de 8 a $14 \mathrm{~cm}$, con lámina acorazonada, con margen liso. Las flores son muy llamativas, miden 6 a $9 \mathrm{~cm}$ de diámetro; los sépalos son blanquecinos 0 amarillentos. El fruto es ovoide, la cáscara es dura, amarilla con frutos blancos y oscuros.

El epicarpio es duro, el mesocarpio es blanco y esponjoso de $5 \mathrm{~mm}$ de espesor, lo que favorece al almacenamiento y transporte a largas distancias. Las semillas son planas, negras, elípticas, rodeadas por un arilo transparente que es la parte 
comestible. Se conocen algunas variedades.

\section{Variedades a utilizar}

- Amarilla

- Colombiana

La propagación de la granadilla se realiza por semilla y esquejes maduros (ramas).

La siembra se realiza en el mes de setiembre si se dispone de agua de riego para el almácigo y al comienzo de las lluvias en caso contrario.

Las semillas se separan de la pulpa mediante la utilización de tamices de malla con diámetro menor al de la semilla, luego se lavan con bastante agua y luego se extienden sobre papel periódico, el secado se efectúa a la sombra.

Cantidad de semillas por Ha:

Se utiliza 0.5 kilogramo de semilla para una hectárea de cultivo.

Germinación de 20 a 30 días.

Hurtado (2008). Menciona que el almácigo puede hacerse en camas 0 en bolsitas. Si se hace en camas, la siembra puede efectuarse en surquitos distanciados $5 \mathrm{~cm}$ y la semilla cada $2 \mathrm{~cm}$; la semilla se pone a un centímetro de profundidad: los riegos deben ser frecuentes.

Cuando las plantitas tienen 20 a $30 \mathrm{~cm}$ de alto están listas para el trasplante a campo definitivo, para ello se surquea y se hacen hoyos.

Distancia entre surcos: $3.00 \mathrm{~m}$, entre hoyos $1.50 \mathrm{~m}$, hacer hoyos de $20 \times 20 \mathrm{~cm}$ y $25 \mathrm{~cm}$ de profundidad

El almácigo en bolsitas es mejor porque las plantitas en el trasplante sufren menos, se usan bolsas de $10 \times 15 \mathrm{~cm}$, con 4 huecos hechos a $4 \mathrm{~cm}$ de la base para el drenaje del ceso de agua de los riegos; la siembra se hace a razón de 3 semillas distanciadas entre si y a $1 \mathrm{~cm}$ de profundidad. Las bolsas se alienan en la cama del almácigo formando líneas y columnas. Antes de llevarlas al campo definitivo, es necesario realizar el almácigo, sean estas de semillas 0 esquejes

\section{Colocación de postes y alambres para las espalderas a lo largo de los surcos}

Los postes pueden ser de cualquier planta que no se pudra rápidamente, deben ser de 4 a 4 metros de alto y de 10 a $12 \mathrm{~cm}$ de diámetro, la base de cada de cada poste puede ser embreada unos $50 \mathrm{~cm}$. para aumentar su duración al evitar su pudrición. Estos postes se colocan en huecos en línea recta y al costado de cada surco.

Colocados los postes, se tiende una línea de alambre que une las cabezas de los postes, este alambre de preferencia del $\mathrm{N}^{0} 12$, a $70 \mathrm{~cm}$, más abajo se tiene otra línea de alambre que puede ser más delgado, del $N^{0} 16 ;$ y $100 \mathrm{~cm}$ más abajo una tercera línea de alambre o cordel de plástico, pues solo sirve para sostener la planta durante su primera edad.

A medida que las ramas de granadilla van creciendo se las va ayudando y guiando para que sus zarcillos se prendan en las líneas de alambre en forma de que queden extendidas como un abanico, hasta que alcancen la línea del alambre superior, en este momento ese alambre comenzará a sostener todo el peso de la planta de granadilla, que puede llegar a pesar junto con la fruta hasta $30 \mathrm{~kg}$.

La granadilla responde bien al deshierbo, abonamiento nitrogenado y fosfatado.

El sistema de plantación está determinado por las características del terreno. En el caso de terrenos con pendiente, el trazo debe hacerse en sentido transversal a la pendiente, abriendo las curvas de nivel a la construcción de terrazas; y en terrenos rocosos mediante la apertura de 
hoyos individuales para cada planta al igual que en terrenos planos.

De acuerdo a la configuración del terreno se puede realizar plantaciones en:
a. cuadrado
b. rectangular
c. Tresbolillo

\section{Épocas de trasplante}

La más propicia corresponde a los meses de precipitación pluvial.

Las plantas que son instaladas en los meses de octubre a enero desarrollan mejor su sistema radicular, en relación a las que se instalan en otras épocas del año.

La extracción de las plantitas del almácigo se hace cuidadosamente evitando la rotura de raíces y se llevan al campo en balde para que no sufran de agua. En cada hoyo se coloca 1 ó 2 plantitas separadas y se echa tierra de la mejor calidad que se disponga en el lugar.

Es recomendable hacer el trasplante en días nublados 0 en su defecto muy temprano en la mañana o pasado las 4 de la tarde. El riego se efectúa inmediatamente terminado el trasplante, el que debe ser cuidadosamente vigilado.

Distanciamiento a utilizarse $3.00 \mathrm{~m}$ entre surcos $\mathrm{x}$ $1.50 \mathrm{~m}$ entre plantas.

\section{Plantación propiamente dicha}

Se abren hoyos individuales de $20 \times 20 \times 25 \mathrm{~cm}$, a los cuales se agrega de 2 a $4 \mathrm{~kg}$ de materia orgánica y fertilizantes como abono; aislándolo de cuando se utilizan las curvas de nivel conviene incorporar abono en la misma proporción que la anterior y a surco corrido.

La poda del segundo año, cuando la planta ya produce, es de formación, la eliminación de las ramas mal orientadas evita el excesivo crecimiento longitudinal y abaratan la futura cosecha, facilitando de esta manera el paso de maquinarias e instrumentos en los terrenos con plantaciones y fundamentalmente da facilidad al cosechador.

Las podas severas retrasan el desarrollo vegetativo de las plantas. Después del tercer año, la poda está orientada a eliminar los brazos caducos o defectuosos, principalmente las que ya fructifican poco, porque la tendencia mayoritaria de la tuna es producir más frutos en los cladodios que brotan en la primavera y menos en las de dos o mayores años, por lo que es necesario eliminarlas. Esta labor también se realiza en los frutos.

Los injertos tiene dos objetivos: primero, encontrar el tipo de injerto más adecuado para mejorar la variedad de la granadilla, y el segundo, evaluar el desarrollo y la velocidad de crecimiento de los injertos realizados.

La floración dura aproximadamente 5 meses, de octubre a enero.

\section{Fertilización}

Se utiliza la fórmula 60-50-0 de NPK/Ha con resultados positivos, debiéndose realizar estudios para determinar y precisar sus requerimientos óptimos. La época adecuada para realizar es antes del brotamiento de botones y/o nuevas hojas. Se realiza utilizando 4 puntos equidistantes en la banda de la proyección de la copa, en hoyos bastante superficiales.

Para no dañar la raíz que se extiende por todo el suelo en un nivel no muy profundo. La aplicación de nitrógeno incrementa el número de hojas, siendo esta acción la más útil para incrementar el área de producción de frutos por cada planta. 


\section{Cosecha}

Las plantas bien llevadas no deben pasar de 1.80 metros de altura. El recojo se realiza cuando maduran y una por una, en canastas especiales, luego vendrá la limpieza de los frutos. Posteriormente se realiza la clasificación por tamaño y sanidad, llenándolos en cajones.

La primera cosecha empieza a los 9 a 10 meses después del trasplante y puede prolongarse durante 3 a 7 años, según sea las condiciones del clima, suelo y cuidados que se le dé.

La cosecha se realiza juntamente con su pedúnculo que se corta con tijera podadora 0 cuchillo lo cual ayuda al manipuleo. Luego se realiza la selección y clasificación por el tamaño, sanidad, peso y coloración.

Para el transporte se utilizan canastas y depósitos especiales de embalaje de $12 \mathrm{~kg}$ de capacidad, evitando la compactación y aplastado de los frutos, malogrando la apariencia del fruto

Rendimiento fluctúa de 7, 000 a 16,000 kg/Ha.

\section{Otras labores}

Se realiza otras labores como el recalce, control fitosanitario, labores culturales.

\section{VALOR NUTRICIONAL DE LA GRANADILLA}

Cuadro $\mathrm{N}^{\circ} 4$ Composición nutricional de la granadilla

\begin{tabular}{ccc}
\hline Componentes & $\begin{array}{c}\text { Contenido de 100 } \\
\text { g de parte } \\
\text { comestible }\end{array}$ & $\begin{array}{c}\text { Valores diarios recomendados } \\
\text { (basado en una dieta de 2000 } \\
\text { calorías) }\end{array}$ \\
\hline Agua & $86 \%$ & \\
Proteínas & $1.1 \%$ & $300 \mathrm{~g}$ \\
Carbohidratos & $11.6 \%$ & \\
Cenizas & $0.9 \%$ & $66 \mathrm{~g}$ \\
Grasa total & $0.1 \%$ & \\
Calorías & 46 & $25 \mathrm{~g}$ \\
Fibra & $0.3 \mathrm{~g}$ & $60 \mathrm{mg}$ \\
Ácido ascórbico & $20 \mathrm{mg}$ & $162 \mathrm{mg}$ \\
Calcio & $7 \mathrm{mg}$ & $125 \mathrm{mg}$ \\
Fósforo & $30 \mathrm{mg}$ & $18 \mathrm{mg}$ \\
Hierro & $0.8 \mathrm{mg}$ & $20 \mathrm{mg}$ \\
Niacina & $2.0 \mathrm{mg}$ & $1.7 \mathrm{mg}$ \\
Riboflavina & $0.1 \mathrm{mg}$ & \\
\hline
\end{tabular}

Fuente: Horticulture Perdue (USA) - New Crops

\section{MATERIAL Y MÉTODOS}

Se trabaja con una metodología de orden matemático para el procedimiento de las proyecciones comerciales y el empleo de Soware de Matcad-2, es decir que se procede para la estimación de datos trabajando con la estimación de mínimo cuadrático, donde se obtienen los valores observados y estimados. Para procesar y determinar las curvas de indiferencia comercial se trabaja con las funciones de punto y de curva para estimar el proceso de estandarización de mercado tal como se determina por: Sea una función $y=f(x)$ y $x 0$ un punto del eje $X$, expresado en la función de $h$ la tendencia será un valor positivo 1.

El método a utilizar en el presente trabajo de 
investigación será el inductivo deductivo, método que se halla indisolublemente vinculados entre sí. El proceso de razonamiento deductivo está condicionado por la actividad cognoscitiva así como de la práctica de campo que realizamos en el proceso de la investigación. Este método nos va permitir sistematizar el material empírico que se recopile en la realidad de las microempresas, a partir de las cuales deducir e interpretar el problema de las microempresas dedicadas al cultivo y explotación del cultivo de granadilla.

-Si $h>0, \lim _{\substack{h \rightarrow 0 \\ h>0}} \frac{f(0+h)-f(0)}{h}=\lim _{\substack{h \rightarrow 0 \\ h>0}} \frac{h-0}{h}=1$

Para este caso el principio de los mínimos cuadrados radica en que los valores de análisis deberán escogerse de tal forma que se hagan a $\sum \mathrm{e} 2$ lo más pequeña posible. Una condición necesaria es que las derivadas parciales de la suma con respecto a la propuesta comercial deberán ser iguales a cero con máxima tolerancia de productividad igual al grado marco exportador a) Autores: 3.1. Lugar de ejecución.

El presente trabajo de investigación se llevó a cabo en la provincia de Oxapampa, distritos de Oxapampa y Chontabamba.

\section{Ubicación:}

Oxapampa geográficamente se halla ubicada a 1200 msnm, Latitud Sur $12^{\circ} 30^{\circ} 00^{\prime \prime}$, Longitud Oeste $75^{\circ} 34^{\prime} 05^{\prime \prime}$.

\section{Método de investigación}

El método utilizado en el presente trabajo de investigación fue el inductivo deductivo. El proceso de razonamiento deductivo está condicionado por la actividad cognoscitiva así como de la práctica de campo que realizamos en el proceso de la investigación. Este método nos va permitir sistematizar el material empírico que se recopile en la realidad de las microempresas, a partir de las cuales deducir e interpretar el problema de las microempresas dedicadas al cultivo y explotación del cultivo de granadilla.

\section{RESULTADOS Y DISCUSIÓN}

\section{Variables introducidas/eliminadas (b)}

\begin{tabular}{cccc}
\hline Modelo & $\begin{array}{c}\text { Variables } \\
\text { introducidas }\end{array}$ & $\begin{array}{c}\text { Variables } \\
\text { eliminadas }\end{array}$ & Método \\
\hline 1 & VAR00001, & Análisis & Introducir \\
& VAR0000100(a) & económico. & $\begin{array}{c}\text { Valor variable } \\
\text { predicativa y no } \\
\text { predictiva }\end{array}$ \\
\hline
\end{tabular}

a Alcanzado límite de tolerancia $=100$.

b. Variable dependiente: VAR00001

Resultado № 1 ; Análisis de varianza para 4 años de predicción económica de granadilla a precios exportables cod. 5890-CM-MIN

\begin{tabular}{|c|c|c|c|c|c|c|}
\hline & \multicolumn{6}{|c|}{ Valor de prueba = 0} \\
\hline & \multirow[t]{2}{*}{ t } & \multirow[t]{2}{*}{ gl } & \multirow{2}{*}{$\begin{array}{l}\text { Sig. } \\
\text { (bilat } \\
\text { eral) }\end{array}$} & \multirow{2}{*}{$\begin{array}{l}\text { Diferencia } \\
\text { de } \\
\text { medias }\end{array}$} & \multicolumn{2}{|c|}{$\begin{array}{c}95 \% \text { Intervalo de } \\
\text { confianza para la } \\
\text { diferencia }\end{array}$} \\
\hline & & & & & Inferior & Superior \\
\hline VAR00001 & 4.024 & 3 & 0.028 & 8.75 & 1.8292 & 15.6708 \\
\hline VAR00004 & 8.693 & 3 & 0.003 & 11.5 & 7.29 & 15.71 \\
\hline VAR00006 & 5.902 & 3 & 0.01 & 9.75 & 4.4925 & 15.0075 \\
\hline
\end{tabular}

\section{Prueba para una muestra}

Resultado № 2. Se puede inferir que entre los precios existe para los precios exportables valor real entre los límites inferior y superior 
Resultado № 3. Grafico de Pareto:

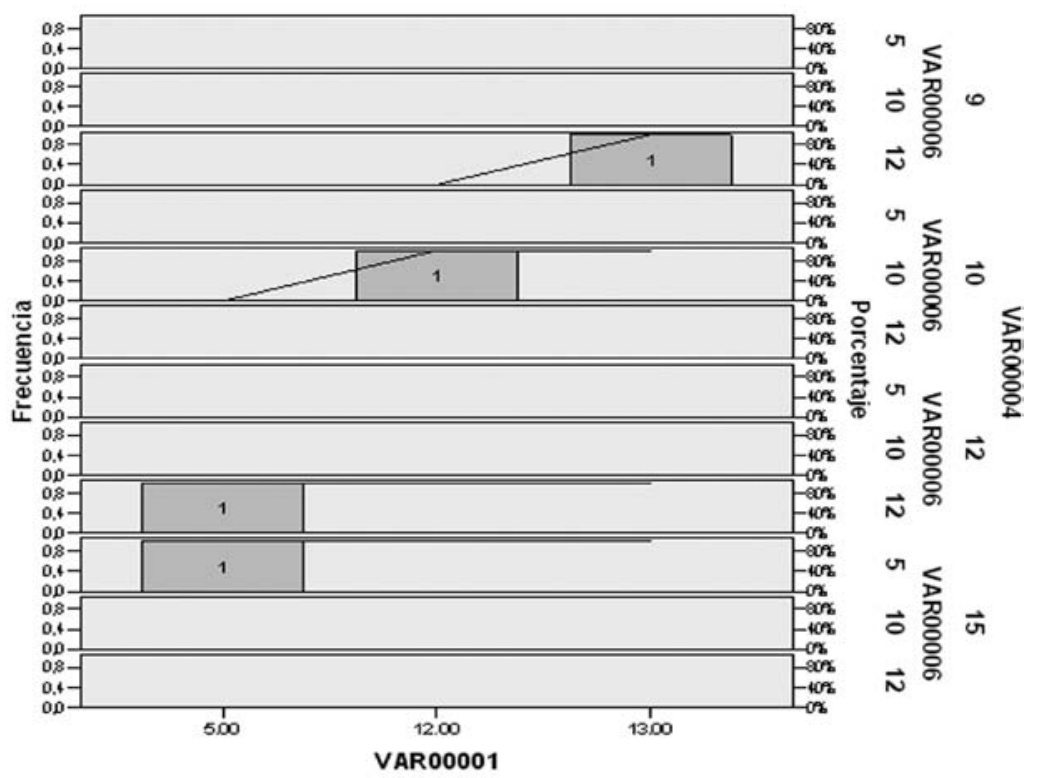

Se aprecia que dentro del control porcentual comercial las secuencias de precio 5 y 12 son las mas constantes lo que muestra entre las frecuencias 0.4 y 0.8 disponibles para valores de promoción como se aprecia en el cuadro.
Para los demás años las predicciones casi serán continuas aun con estas variables se recomendaría tomar predicciones después de 10 años lo que se mostrará en cuadros. Esta proyección se da máximo casi con los 2,5 años y con precio en soles de 7.

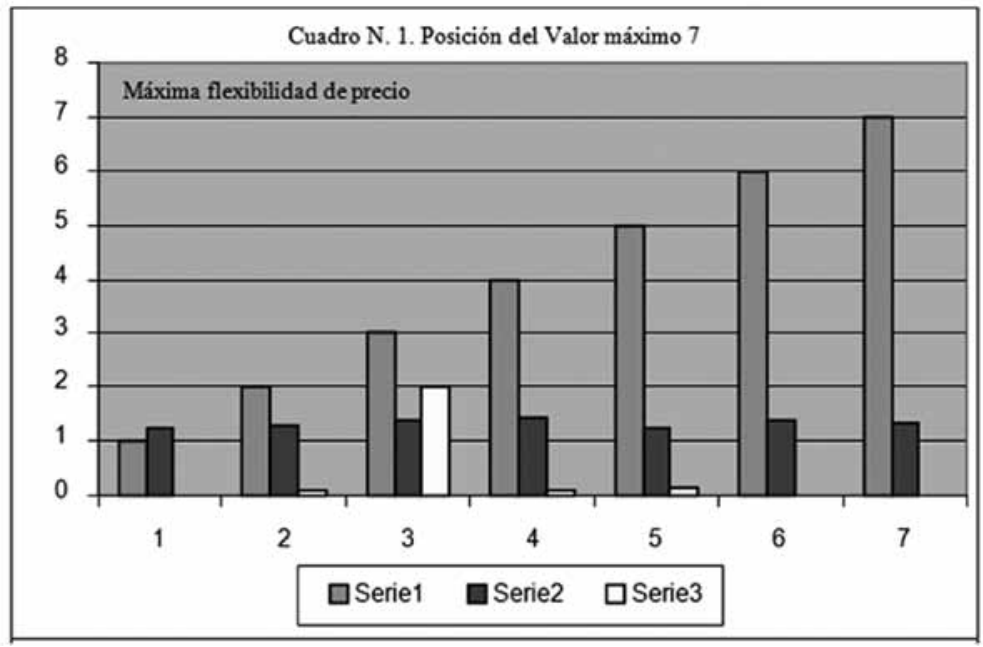

Del Cuadro $\mathrm{N}^{0} 1$. se aprecia la máxima flexibilidad en precio del mercado de granadilla 


\section{Prospectiva Universitaria}

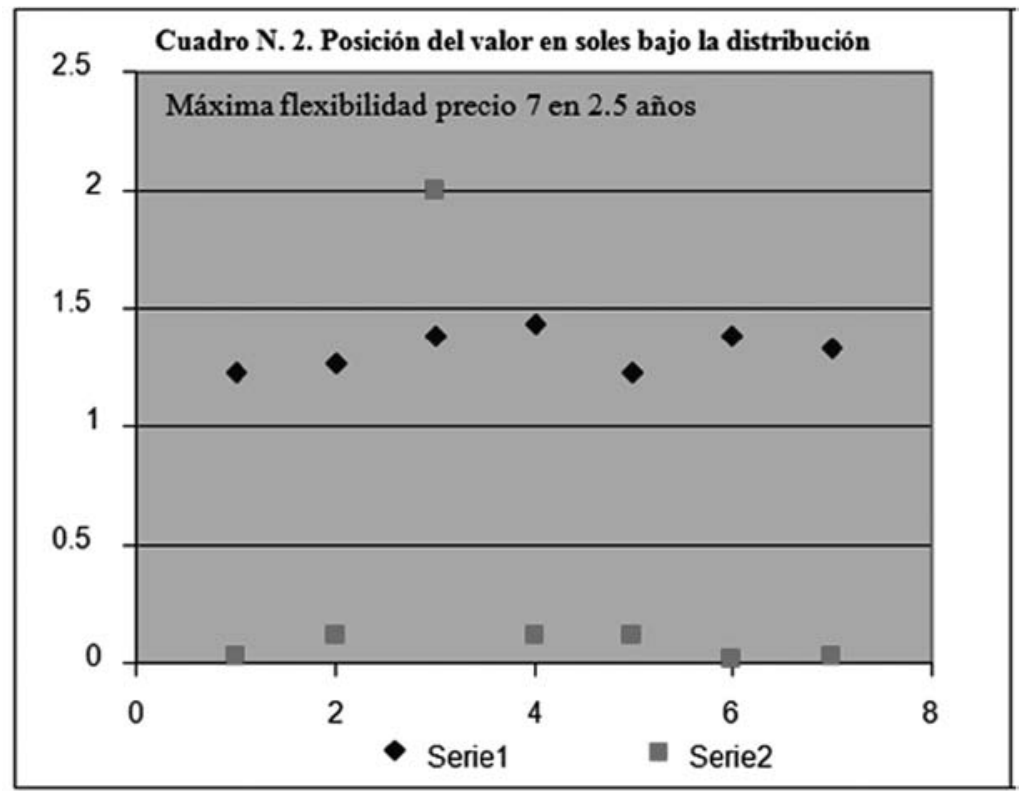

Del Cuadro $\mathrm{N}^{\circ} 2$. El máximo valor comercial del valor de venta de granadilla en un tiempo esperado de 2.5 años perfecto nivel alcanzando su amplitud antes de los 4 años como se muestra en el anova.

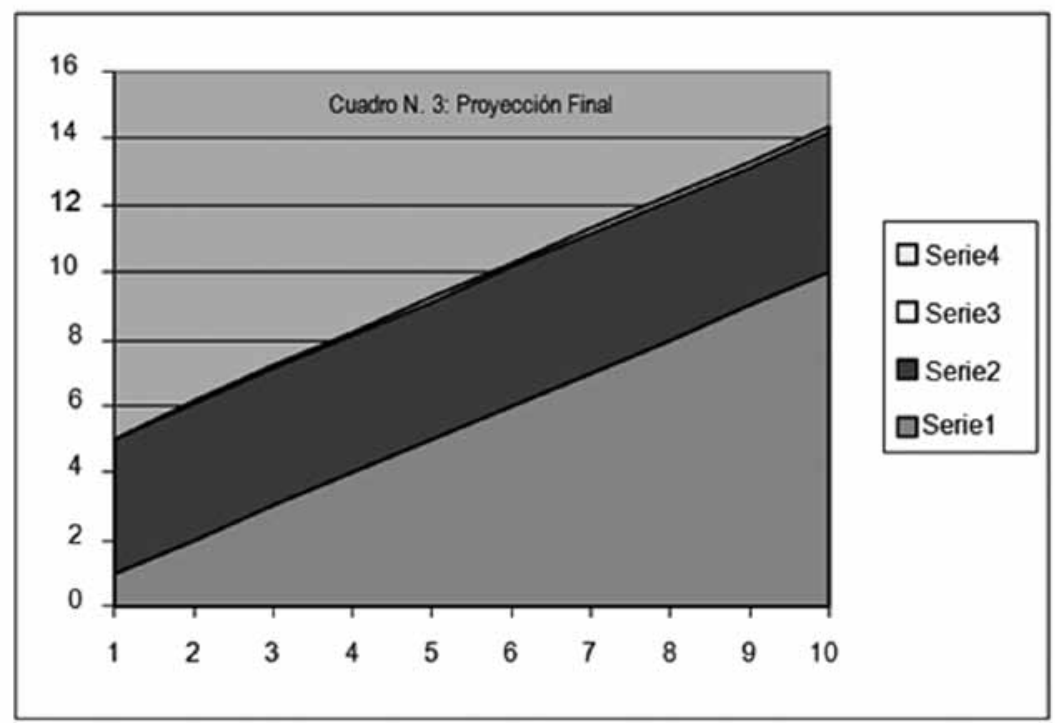

Del Cuadro № 3 . Se muestra la proyección se desearía alcanzar para próximos años con valores máximos alcanzados. 


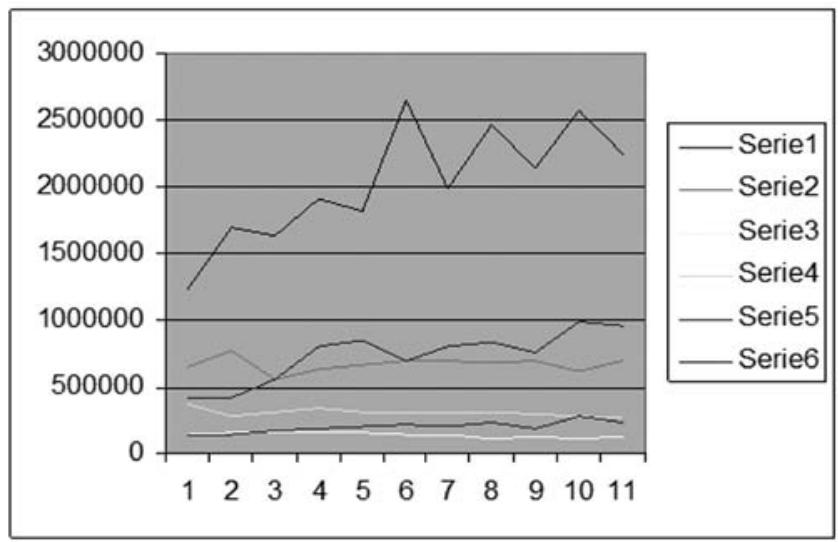

Del Cuadro $N^{\circ} 4$. Del cuadro comparativo se estima para el valor comercial el valor proximal en los próximos años tendrá un valor importante ya que la gradiente de comparación pico alto de 2500000 millones de plantones por superficie incrementan el valor productivo desde un proximal de 500,000 plantones por superficie cultivada.

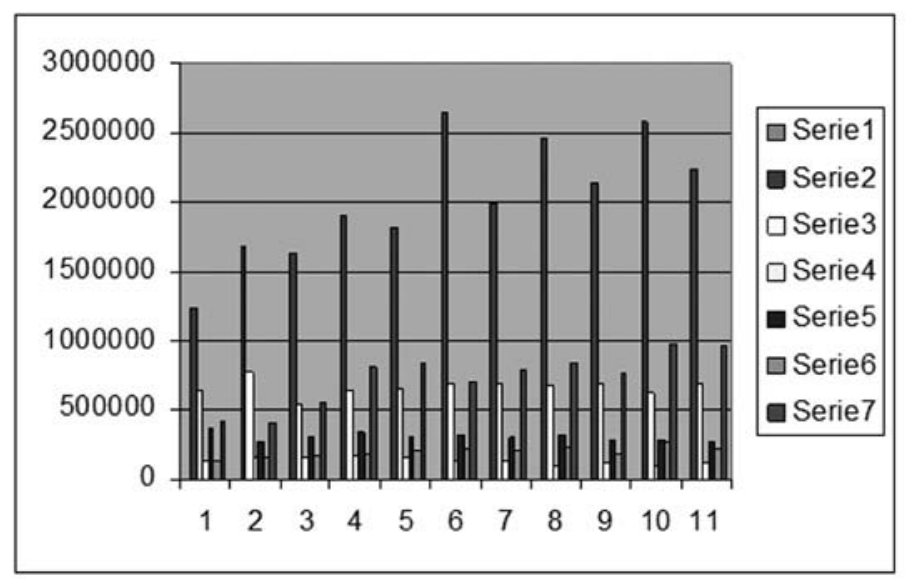

Del Cuadro $N^{0} 5$. La estimación más alta de los picos por superficie cultivada comparada al valor más pequeño lo que indica el incremento productivo, está a su vez supera las condiciones de trabajo y la oportunidad del cambio económico en la región.

Del trabajo desarrollado se llegó a las siguientes conclusiones:

Se debe aperturar con más frecuencia estudios 0 experimentos en el cultivo de la granadilla.

Se debe generar más espacios de explotación de granadilla en nuestra selva central y trabajar con las técnicas modernas de generar economías de escala.

De los cuadros estadísticos se aprecia que es importante para su comercialización mundial aparentemente se puede generar más 
comunicación con espacios de exportación. REFERENCIAS BIBLIOGRÁFICAS

Se debe consolidar la generación de nuevas Jiménez Coz, José. Manejo e importancia de la expectativas de reconversión productiva y granadilla en el Perú. Lima Peru.1ra. Edición. mejorar las estadísticas de producción. Editora.Minag. 2006.

Consolidar la importancia de la granadilla para Hurtado Cabieses, Pedro. Propuestas efectos de introducir una explotación comercial a económicas para el Manejo de Granadilla en gran escala para tratar de la importancia de este zonas productoras de Selva Peruana. Lima fruto en el mundo

Peru.1ra Edición Ediciones Perú Agro. 2008. 